\title{
Application of control methods for modelling the flow of Pine Island Glacier, West Antarctica
}

\author{
Andreas VIELI, Antony J. PAYNE \\ Centre for Polar Observation and Modelling, School of Geographical Sciences, University of Bristol, University Road, Bristol BS8 1SS, England \\ E-mail:a.vieli@bristol.ac.uk
}

\begin{abstract}
The distribution of basal traction on a transect along Pine Island Glacier, West Antarctica, is estimated by inverting observed surface velocities using a control method and a simple numerical stream-flow model. This model calculates the horizontal flow along a transect, based on the assumptions that the horizontal flow is independent of ice depth and that the driving stresses are balanced by resistive forces at the glacier bed and margin and by gradients in longitudinal stress. Basal traction is assumed to be linearly related to the basal velocity. For the lateral shear traction a parameterization based on an inversion of Glen's flow law is used. The application of the control method allows us to calculate the set of model parameters (e.g. the basal friction coefficient) that gives the best fit between modelled and observed surface velocities. The model is used to investigate the stress regime of Pine Island Glacier, in particular to estimate the importance of basal, lateral and longitudinal stresses relative to each other. In the flat region just behind the grounding line, basal drag, lateral drag and the longitudinal stress gradient are the same order of magnitude. In the steep region up-glacier from the grounding line, the driving stresses are highest and balanced predominantly by basal resistive stresses. Further upstream, in the trunk of the glacier, lateral and basal drag predominate.
\end{abstract}

\section{INTRODUCTION}

Pine Island Glacier (PIG, Fig. 1) has the largest discharge of all West Antarctic ice streams $\left(66 \mathrm{Gt} \mathrm{a}^{-1}\right)$. Although the mass balance of the Pine Island drainage basin is close to zero (Vaughan and others, 2001), satellite observations show a significant grounding-line retreat of PIG of about $1.2 \mathrm{~km} \mathrm{a}^{-1}$ between 1992 and 1996 (Rignot, 1998), and thinning of the grounded terminus by up to $1.6 \mathrm{~m} \mathrm{a}^{-1}$ between 1992 and 1999 (Shepherd and others, 2001). This thinning cannot be explained by short-term variations in accumulation and is expected to be a result of a change in the dynamics of the glacier. Whether this change is a consequence of external forcing or internal feedbacks is not yet clear. An improved understanding of the controls on the flow of PIG is needed to assess the causes of the observed changes.

In the main trunk of PIG, driving stresses are rather low $(\sim 30 \mathrm{kPa})$, whereas directly behind the grounding line high driving stresses $(>100 \mathrm{kPa}$ ) occur (Vaughan and others, 2001). The high driving stress and a significant deepening (approximately $500 \mathrm{~m}$ ) of basal topography inland of the grounding line are in contrast to the low driving stresses $(\sim 10 \mathrm{kPa})$ and flat base of the ice streams in the Siple Coast area of West Antarctica. PIG does, however, have shear margins bordering slow-moving ice, which are characteristic of other West Antarctic ice streams and suggest that resistive forces from the margins may be an important control on the flow. Ice-penetrating radar data suggest that the base is at the pressure-melting point in the main trough (Vaughan and others, 2001).

The aim of this study is to investigate the flow and stress regime of PIG, and in particular to evaluate the role of basal and lateral drag, and longitudinal stresses in the force balance. A special focus is given to the determination of the unknown basal friction field. A numerical model for ice-stream flow is used to calculate the flow of PIG along a flowline. The basal friction distribution is estimated by inverting observed surface velocities using a control method, which is presented in detail in this study. The control method used here is similar to that applied by MacAyeal and others (1995) on Ice Stream E.

\section{FLOW MODEL}

The numerical flow model used in this study calculates the horizontal flow along a flowline ( $x$ direction). It is based on a leading-order approximation of the stress equilibrium equations for ice-stream and shelf flow derived by MacAyeal (1989). The main assumption is that vertical shearing of the horizontal velocity $u$ is negligible, which means the horizontal flow is independent of the vertical coordinate $z$.

The high driving stresses in the main trunk behind the grounding line (315-365 km in Fig. 3c and h, shown later) imply that here vertical shear of horizontal velocity may become important. Using the shallow-ice approximation (Hutter, 1983), the horizontal velocity due to vertical shearing is estimated on the basis of surface slope and ice thickness. A temperature-dependent rate factor is used (Paterson and Budd, 1982), and as a first approximation the vertical temperature profile is assumed to increase linearly from the mean surface air temperature $T_{\mathrm{s}}=-22^{\circ} \mathrm{C}$ (Giovinetto and others, 1990) to $T_{\mathrm{b}}=0^{\circ} \mathrm{C}$ at the bed. This estimated deformation velocity is in most parts well below $3 \%$ of the observed surface velocities and below $17 \%$ in the region where the high 
driving stresses occur (Fig. 3b and g, shown later). Furthermore, most of the deformation is expected to occur in the basal layers (perhaps with enhancement due to fabric evolution) and therefore the horizontal velocity will be more-orless constant for most of the ice thickness. Omitting vertical shear in our model is therefore a reasonable first approximation. The one problematic area is where the high driving stresses occur $(325-365 \mathrm{~km})$. However, whether we take the velocity constant with depth or not is expected to have little effect on the basal drag (Van der Veen and Whillans, 1989; Whillans and others, 1989). We will return to this assumption of no vertical shear in the discussion, because a detailed analysis would require knowledge of the lateral and basal traction, which we do not have at this stage but which will be available after our analysis.

In this study, only horizontal flow $u$ along a flowline is considered ( $x$ direction), and we assume there is no flow in the transverse direction to the ice stream ( $y$ direction). The force balance in a profile along the flowline is then given by

$$
-2 \frac{\partial h \sigma_{x}^{\prime}}{\partial x}-\frac{\partial h \tau_{x y}}{\partial y}+\tau_{\mathrm{b}}=-\rho g h \frac{\partial s}{\partial x},
$$

where $h$ is the ice thickness, $\sigma_{x}^{\prime}$ is the longitudinal stress deviator defined as the difference between the longitudinal stress and hydrostatic pressure, $\tau_{x y}$ is the lateral shear stress, $\tau_{\mathrm{b}}$ the shear traction at the bed, $\rho$ the density of ice, $g$ the acceleration due to gravity and $s$ the surface elevation. Thus the driving stress $\tau_{\mathrm{d}}$ on the righthand side of Equation (1) is balanced by gradients in longitudinal stress and resistive forces at the glacier bed and at the margins. Using Glen's flow law (Glen, 1955) and defining an effective viscosity $\nu=A^{-1 / n} \dot{\varepsilon}^{(1-n) / n}$, where $n$ is the flow-law exponent, $A$ the rate factor and $\dot{\varepsilon}$ the effective strain rate, $\sigma_{x}^{\prime}$ can be written as

$$
\sigma_{x}^{\prime}=\nu \frac{\partial u}{\partial x}
$$

The effective strain rate is calculated assuming no vertical and lateral shearing of the horizontal velocity, which leaves the longitudinal variation alone and leads to an underestimation of $\dot{\varepsilon}$ in places where the longitudinal strain rates are close to zero.

The basal shear traction of the ice stream is assumed to be linearly related to the basal velocity $u_{\mathrm{b}}$ (MacAyeal, 1989):

$$
\tau_{\mathrm{b}}=\beta^{2} u_{\mathrm{b}},
$$

where $\beta^{2}$ is the basal friction coefficient. A realistic relationship between $\tau_{\mathrm{b}}$ and $u_{\mathrm{b}}$ is likely to be non-linear, but the details of such non-linearities are unknown (Raymond, 1996). Therefore the most simple case of a linear relation is considered here. At the base of the ice shelf, basal traction $\tau_{\mathrm{b}}$ is zero which is included in the model by setting $\beta=0$.

Because resistance to flow from side shear is recognized to be important in the force budget of ice streams (Echelmeyer and others, 1994), lateral drag is included in the model using a parameterization suggested by Van der Veen and Whillans (1996). Integration of Equation (1) over the width of the ice stream, assuming constant ice thickness across the channel of half-width $W$, gives

$$
-2 \frac{\partial h \sigma_{x}^{\prime}}{\partial x}+\frac{h}{W} \tau_{\mathrm{s}}+\tau_{\mathrm{b}}=-\rho g h \frac{\partial s}{\partial x}
$$

where $\tau_{\mathrm{S}}$ is the lateral stress. Considering lateral drag independent of basal drag and linking the lateral shear stress to

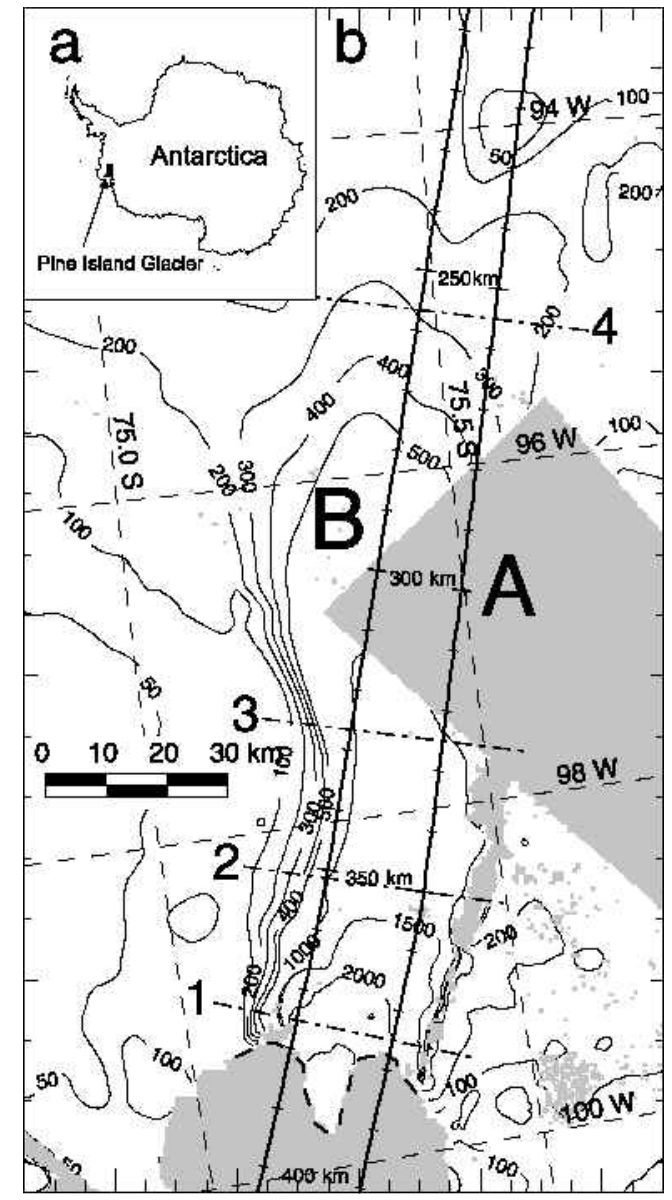

Fig. 1. Horizontal surface velocities of Pine Island Glacier derived from satellite interferometry. Contour intervals are $100 \mathrm{ma}^{-1}$ below $500 \mathrm{ma}^{-1}$, and $500 \mathrm{ma}^{-1}$ above. The thick solid lines show the two transect profiles for which the basal topography is known and to which the model is applied. The kilometre marks refer to the distance along the profiles used in Figures 2-6. The dashed-dotted lines indicate the locations of the cross-profiles shown in Figure 2. The thick dashed line indicates the grounding line. In grey areas no velocity observations are available, because of either missing satellite data coverage or loss of coherence.

the transverse shear strain rate with Glen's flow law, $\tau_{\mathrm{s}}$ can be expressed in terms of the width-averaged velocity $\bar{u}$ by

$$
\tau_{\mathrm{s}}=\left(\frac{5}{2 A W} \bar{u}\right)^{1 / 3}
$$

An alternative way of including the lateral drag is to calculate the term $\partial h \tau_{x y} / \partial y$ in Equation (1) directly from the observed two-dimensional horizontal surface velocity field, which has been done in some additional model runs. Unless noted differently the parameterization of Van der Veen and Whillans (1996) is used.

Assuming no spreading at the sides and no lateral variation of $u$, the boundary condition at the shelf front derives from the force balance between ice and displaced ocean water (Paterson, 1994, p. 296).

\section{Model equation and solution technique}

Substituting $\sigma_{x}^{\prime}, \tau_{\mathrm{b}}$ and $\tau_{\mathrm{s}}$ into Equation (4) gives the final 
model equation for the width-averaged horizontal velocity $\bar{u}:$

$$
-2 \frac{\partial}{\partial x}\left(\nu h \frac{\partial \bar{u}}{\partial x}\right)+\frac{h}{W}\left(\frac{5}{2 A W}\right)^{1 / 3} \gamma \bar{u}+\beta^{2} \bar{u}=-\rho g h \frac{\partial s}{\partial x}
$$

The lateral drag (second term on lefthand side) is linearized in $\bar{u}$ by defining $\gamma=\bar{u}^{-2 / 3}$. To simplify the notation below, the width-averaged horizontal velocity is denoted by $u$ instead of $\bar{u}$. The model equation (6) is solved numerically using the finite-difference method and iterating for the effective viscosity $\nu$ and $\gamma$. Approximating the $x$ derivatives by centred differences, the discretized model equation can be written as a linear system of equations in the unknown surface velocity $u_{j}$ :

$$
H_{i}=\mathbf{a}_{i j} u_{j}-c_{j}=0 \quad i=1, \ldots, n,
$$

where $i$ and $j$ denote the gridpoint number, $\mathbf{a}$ is a tridiagonal $n \times n$ matrix and is a function of $\nu$ and $\gamma$. For each iteration step, this linear system of equations is solved for $u_{i}$ using $\nu$ and $\gamma$ calculated from the velocities of the previous iteration step.

\section{GONTROL METHOD}

The basal friction coefficient $\beta^{2}$ in Equation (6) is an unknown parameter in our numerical model for ice-stream flow. We determine it here by inverting observed surface velocities using a control method similar to that used by MacAyeal (1993) and MacAyeal and others (1995). For details of this method we refer to applications in oceanography (Thacker, 1988; Thacker and Long, 1988; Schlitzer, 1993).

The control method is designed to find the set of unknown model parameters $\mathbf{p}=\left(p_{1}, \ldots, p_{m_{p}}\right)$ which when substituted into the model equation produces the best fit between modelled $\mathbf{q}=\left(q_{1}, \ldots, q_{m_{q}}\right)$ and observed $\mathbf{q}^{\mathrm{d}}=\left(q_{1}^{\mathrm{d}}, \ldots, q_{m_{q}}^{\mathrm{d}}\right)$ quantities. The misfit is measured by a cost function $J$, which is taken here as

$$
J=\sum_{j=1}^{m_{q}} \frac{\left(q_{j}^{\mathrm{d}}-q_{j}\right)^{2}}{\sigma_{q}^{2}}
$$

where $\sigma_{q}^{2}$ is a weighting factor (ideally taken as the observational error). Searching for the set of model parameters $p_{i}$ that gives the best fit is the same as minimizing $J$ while ensuring that the model equations are satisfied. This is done by the method of Lagrange multipliers (Thacker, 1988). The model equation (6) written in the finite-difference form for each gridpoint $i$ is given by

$$
H_{i}(\mathbf{q}, \mathbf{p})=0 \quad i=1, \ldots, n .
$$

We now introduce the Lagrangian $L$ by

$$
L=J+\sum_{i=1}^{n} \lambda_{i} \cdot H_{i}(\mathbf{q}, \mathbf{p}) \quad i=1, \ldots, n,
$$

where $\Lambda=\left(\lambda_{1}, \ldots, \lambda_{n}\right)$ are the Lagrange multipliers. The importance of the Lagrangian is that the minimum of the cost function is found when the gradient of $L$ in respect to $\lambda_{i}, q_{j}$ and $p_{j}$ vanishes:

$$
\begin{array}{ll}
\frac{\partial L}{\partial \lambda_{i}}=H_{i}=0 \quad i=1, \ldots, n & \\
\frac{\partial L}{\partial q_{j}}=\frac{\partial J}{\partial q_{j}}+\sum_{i=1}^{n} \lambda_{i} \frac{\partial H_{i}}{\partial q_{j}}=0 \quad j=1, \ldots, m_{q} \\
\frac{\partial L}{\partial p_{j}}=\frac{\partial J}{\partial p_{j}}+\sum_{i=1}^{n} \lambda_{i} \frac{\partial H_{i}}{\partial p_{j}}=0 \quad j=1, \ldots, m_{p} .
\end{array}
$$

On differentiation with respect to $\lambda_{i}$, Equation (11) recovers the model equation. Seeking for the minimum of $J$ is now equivalent to finding a model solution that satisfies the model equation (9) and the adjoint model equations (12) and (13). Equation (12) represents a system of $n$ linear equations in the $n$ unknown Lagrange multipliers $\Lambda=\left(\lambda_{1}, \ldots, \lambda_{n}\right)$ from which $\Lambda$ can be calculated. Introducing $\Lambda$ into Equation (13) allows us to calculate the gradient of $L$ with respect to the model parameters $p_{j}$ given by $\nabla J=\partial L / \partial p_{j}$. It is down this gradient that a new improved guess for the model parameters $\mathbf{p}$ is determined by applying a descent algorithm (Polak-Ribiere method; Press and others, 1989, p. 303).

In this study, the observed surface flow field is inverted for the basal friction coefficient $\beta$. Thus, the unknown model parameters $\mathbf{p}$ are given by the basal friction coefficient $\beta_{i}$ at each gridpoint $i=1, \ldots, m$ and $\mathbf{q}^{\mathrm{d}}$ and $\mathbf{q}$ are given by the observed and modelled horizontal surface velocities $u_{i}^{\mathrm{d}}$ and $u_{i}$, respectively. The weighting constant $\sigma_{q}$ is taken equal to the observation error of the surface velocity $\left(\sigma_{u}\right)$. The control method algorithm can be summarized as follows. First, the model parameters $\beta_{j}$ are initialized by a first arbitrary guess $\beta_{\text {initial. }}$. The following steps are then repeated until the minimum of $J$ is reached or $J$ is reduced to a value that is consistent with the error in the measurements. The cost function $J$ is evaluated and $\nabla J=\partial L / \partial \beta_{j}$ calculated by solving the adjoint equations (12) and (13). Using this gradient $\nabla J$ and a conjugate gradient method, a new improved set of model parameters $\beta_{j}$ is determined for input to the ice-stream model.

In our case, the discretized version of the model equation is linear in $u_{i}$ (Equations (6) and (7)) and the adjoint equation (12) can be written as

$$
-\frac{2\left(u_{j}^{\mathrm{d}}-u_{j}\right)}{\sigma_{u}^{2}}+\sum_{k=1}^{n} \lambda_{k} \cdot \mathbf{a}_{j k}^{*}=0 \quad j=1, \ldots, m,
$$

where the ${ }^{*}$ denotes the transpose of a matrix. The matrix $\mathbf{a}_{j k}$ is already known from solving the forward model (Equation (7)); thus the determination of the $\lambda_{i}$ does not need any extra effort.

\section{OBSERVATIONS AND MODEL SETTING}

\section{Velocities and geometry}

The surface velocity field for PIG is known from satellite radar interferometry from European Remote-sensing Satellite (ERS) synthetic aperture radar (SAR) data recorded between 1994 and 1996 (Shepherd and others, 2001). There is a gap in the coverage of the interferometric data on the righthand side of the main trough (Fig. 1). The surface and basal topography is only known on two transect profiles (A and B) from airborne radar sounding (Corr and others, 2001; Vaughan and others, 2001). Because the basal topography is not known 


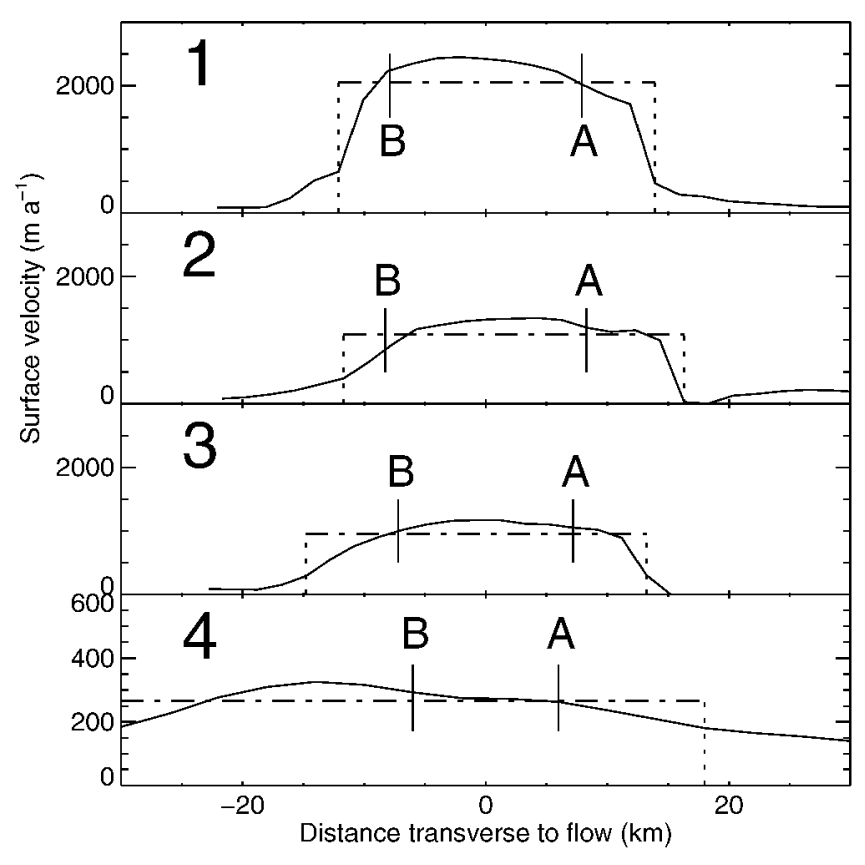

Fig. 2. Surface velocities in the cross-profiles 1-4 marked in Figure 1. The crossings with profiles $A$ and $B$ are marked, and the mean velocities over the stream width are shown as dashed-dotted lines.

outside of these two profiles, we decided to perform the model calculations along the two profiles. Using a transect model for stream flow requires that the profile is approximately along the direction of glacier flow in the lower $200 \mathrm{~km}$ of interest. This is the case for profile A, but, because the glacier turns slightly to the right (in the flow direction) in the fastflowing part, profile B comes close to the ice-stream margin, resulting in a surface velocity depression along this profile (Fig. 1; Fig. 3g, shown later).

\section{Model setting}

The horizontal grid size $\Delta x$ equals $5 \mathrm{~km}$ and the flow-law exponent is set to $n=3$ (Paterson, 1994, p. 85). Unless otherwise specified, a rate factor $A=4.9 \times 10^{-25} \mathrm{~s}^{-1} \mathrm{~Pa}^{-3}$ corresponding to an ice temperature of $T=-10^{\circ} \mathrm{C}$ is used (Paterson, 1994, p.97), and is assumed to be constant over the whole model domain. The flow model calculates the width- and depth-averaged velocity and stress fields for a glacier that flows down a rectangular channel of half-width $W$ and thickness $h$, which both vary along $x$. Instead of observed width-averaged velocities, we used the observed velocities along the profiles and assumed that they are representative for the width-averaged flow. The four velocity crossprofiles shown in Figure 2 support this assumption. The differences between the width-averaged velocities and the velocities at the locations of profiles $\mathrm{A}$ and $\mathrm{B}$ are in general $< \pm 10 \%$.

\section{Glacier width}

The width of PIG along its whole length is not well defined, and a simplified width distribution along the transect is estimated on the basis of the interferometrically derived velocity field (Fig. 1). A half-width of $W=20 \mathrm{~km}$ is chosen for the ice shelf, $W=16 \mathrm{~km}$ for the ice stream in the main trunk and $W=30 \mathrm{~km}$ further up-glacier. In the numerical model, the choice of $W(x)$ only affects the parameterization of the lateral drag (Equation (6)).

\section{Shear softening}

The parameterization for lateral drag used here is based on the assumption that $\tau_{\mathrm{s}}$ is independent of the basal drag and simply linked to the transverse shear strain rate. Thus, the resistance at the margin is expected to be overestimated. Lateral drag may additionally be reduced by softening of the ice in the shear margins due to strain heating and intense crevassing (Echelmeyer and others, 1994). We account for shear softening by introducing an optional shear softening factor $f_{\text {soft }}$ in the parameterization of the lateral drag by

$$
\tau_{\mathrm{s}}=\left(\frac{5}{2 f_{\mathrm{soft}} A W} \bar{u}\right)^{1 / 3} .
$$

In the ice shelf $\beta^{2}=0$ and the driving stress is balanced by lateral drag and longitudinal stress gradients. The parameterization for $\tau_{\mathrm{s}}$ turns out to be unrealistic for the ice-shelf region because there the calculated lateral drag partly exceeds the driving stress. This is mainly a result of the high flow velocities and very low driving stresses in the shelf part. To avoid unrealistically high lateral stresses and to obtain ice-shelf velocities of the right order of magnitude, shear softening for the shelf ice is assumed. This study focuses on the flow and stress regime in the ice stream, which is found to be insensitive to the chosen softening factors and boundary conditions in the ice-shelf part.

\section{Initial guess of $\beta^{2}$}

The control method requires an initial guess for the model parameter $\beta^{2}$, which in this study is chosen generally as the explicit solution $\beta^{2}$ of the model equation (6) neglecting the longitudinal stress gradients. To check the sensitivity of our final results to the initial guess, additional inversions with constant initial $\beta^{2}$ of $2.5 \times 10^{9}$ and $10.0 \times 10^{9} \mathrm{Pasm}^{-1}$, respectively, are calculated.

\section{Data uncertainty}

The error estimate for the observed surface velocity $\sigma_{u}=15 \mathrm{~m}$ $\mathrm{a}^{-1}$ (personal communication from A. Shepherd, 2002) includes random measurement errors and interpolation errors. In a $30 \mathrm{~km}$ section of profile A, the surface velocities are unknown (Fig. 1). Based on the known velocity field in the surrounding areas and along the other transect, we do not expect any unusual changes in the velocity field, and therefore the velocities are linearly interpolated. To account for the additional uncertainty in this region, a larger error of $\sigma_{u}=60 \mathrm{~m} \mathrm{a}^{-1}$ is used.

\section{Acceptance of fit}

A fit between model results and observations, and the associated model parameters, is accepted when the cost function $J$ is reduced to a value $J_{\text {a }}$ consistent with the measurement error in the observations. Assuming that the observation error is a random variable and has a Gaussian probability distribution, our misfit $J$ is closely related to a $\chi^{2}$ distribution with $m-1$ degrees of freedom (Menke, 1989, p. 32; MacAyeal and others, 1995), where $m$ is the number of observed velocities on gridpoints. We accept an inversion run if $J$ is reduced below the bound $J_{\text {a }}$ which is chosen as the level of $\chi^{2}$ that corresponds to a $10 \%$ probability of occurrence (Menke, 1989). This means that a further improvement of the fit could not be differentiated from the effect of random measurement errors. In our case of 50 degrees of freedom, the value of acceptance is $J_{\mathrm{a}}=63.17$ (Mendenhall and others, 
Profile A
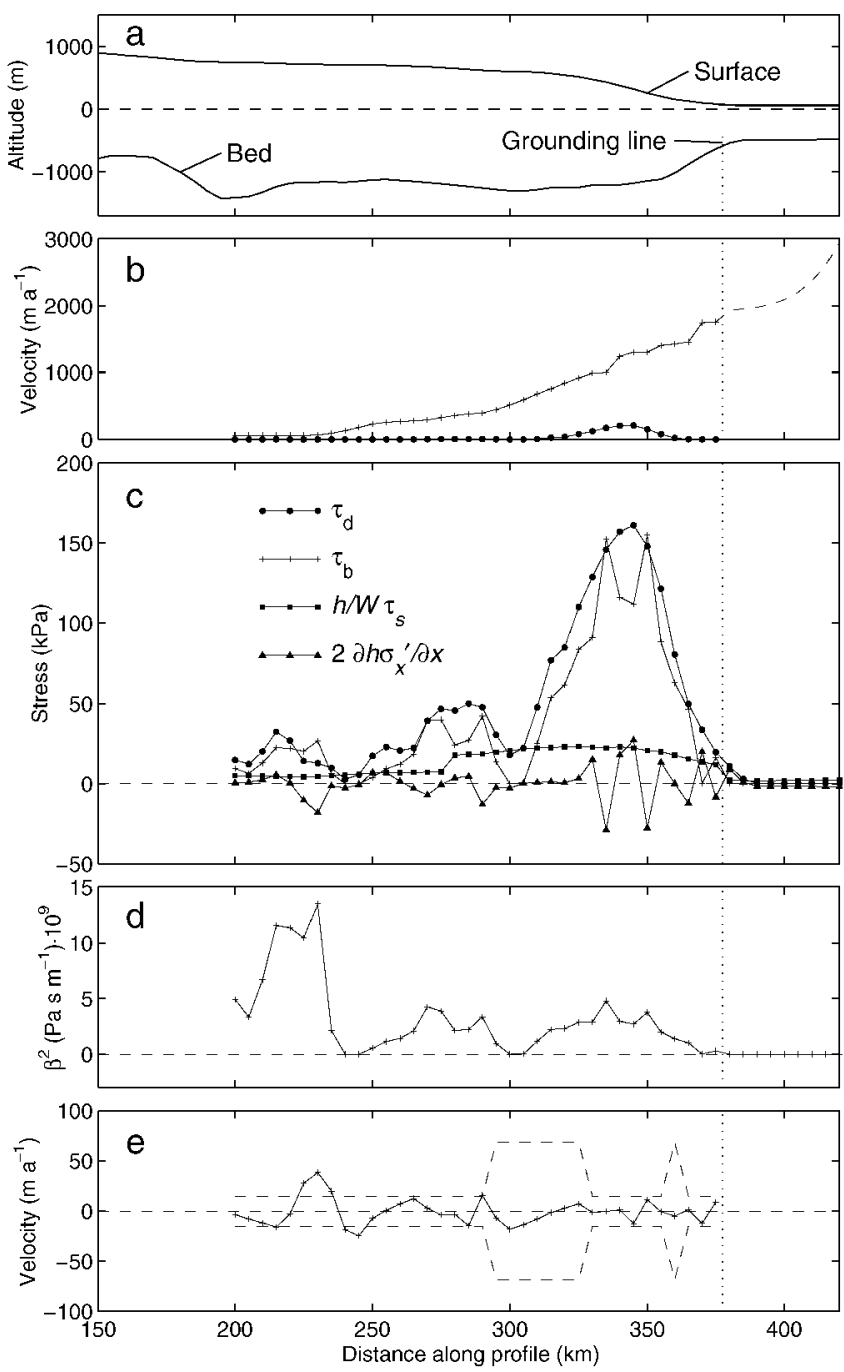

Profile B
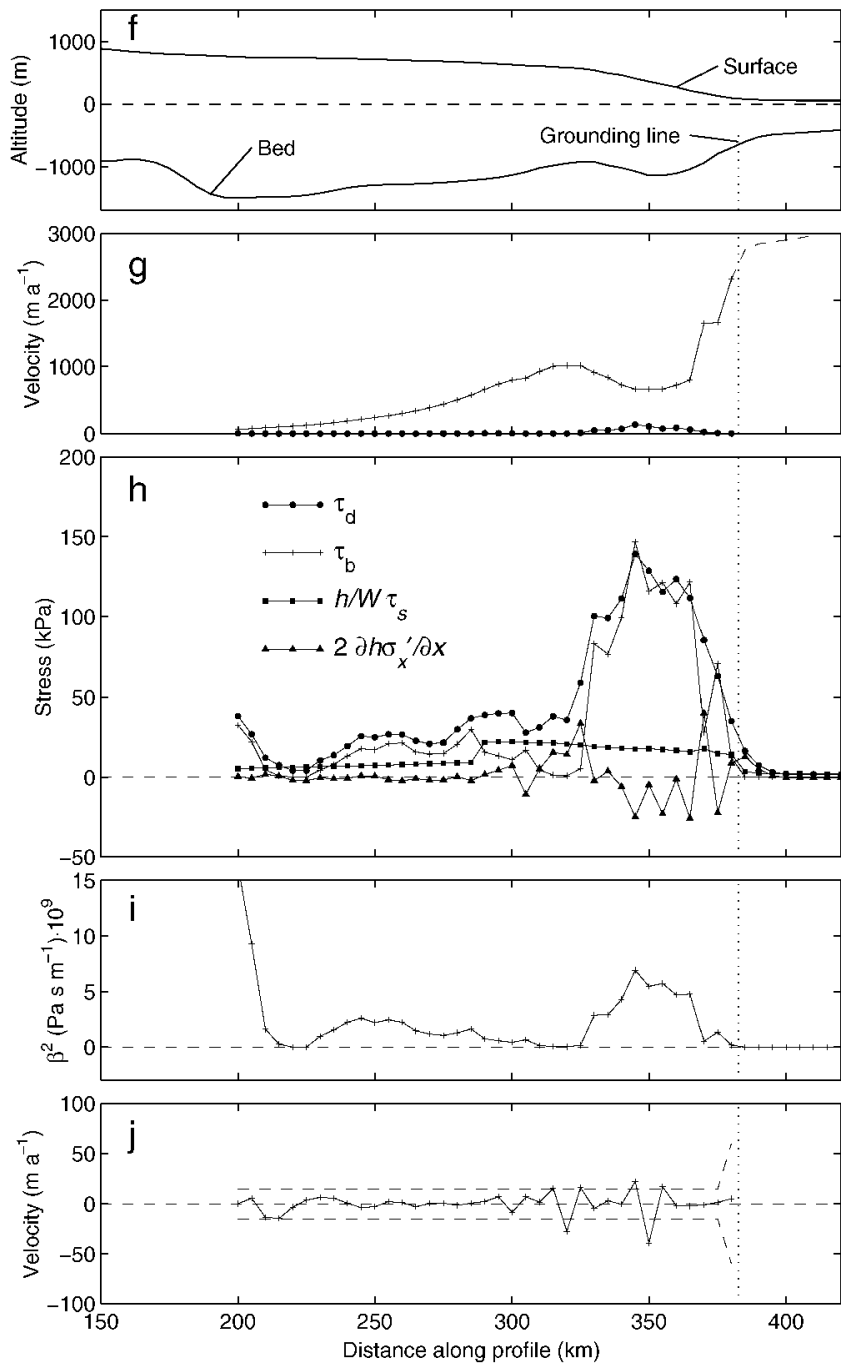

Fig. 3. Profile A: (a) Surface and bed topography; (b) calculated and observed surface velocities (crosses) and estimated vertical shear of the horizontal velocity (dots) using the shallow-ice approximation; (c) driving stress and calculated basal drag $\tau_{\mathrm{b}}$, lateral drag $h / W \tau_{\mathrm{s}}$ and longitudinal stress gradients; (d) calculated basal friction coefficient $\beta^{2}$; and (e) difference between modelled and observed velocities shown with the corresponding measurement error $\sigma_{u}$ (dashed lines). The dotted line here and in the remaining figures indicates the grounding line. Profile B: $(f)$ as for $(a) ;(g)$ as for $(b) ;(h)$ as for $(c) ;(i)$ as for $(d)$; $(j)$ as for $(e)$.

1989). Whenever $J$ falls below this value of 63.17 , the random error in observation could account for a misfit greater than $J$ with a probability of $10 \%$.

\section{MODEL RESULTS}

The model calculations and interpretations are focused on the region where the flow is expected to be dominated by basal sliding, that is, the main trunk and the grounding-line region $(200-380 \mathrm{~km})$.

\section{Reference inversion}

Various runs of the control algorithm were performed for profiles $\mathrm{A}$ and $\mathrm{B}$ with different parameter assumptions. The results of the reference inversions $\mathrm{A}$ and $\mathrm{B}$ are shown in Figure 3. Figure $3 \mathrm{c}$ and $\mathrm{h}$ show each term of our balance equation (6) separately. For the calculated stress regime, three different sections can be identified along the transect for both profiles. In the grounding-line zone and immediately behind (365$385 \mathrm{~km}$, an area often called the ice plain), the resistive stresses from the base and the sides, and the longitudinal stress gradi- ents are all of the same order of magnitude. In the region behind $(315-365 \mathrm{~km})$, the high driving stress $\tau_{\mathrm{d}}$ (up to $160 \mathrm{kPa}$ ), due to a significant increase in surface slope, is mainly balanced by basal drag $\tau_{\mathrm{b}}$. Although lateral drag and longitudinal stress gradients reach the highest values in this region, they are small compared to $\tau_{\mathrm{b}}$. For profile $\mathrm{B}$, the high basal shear traction could be interpreted as a consequence of the local velocity minimum in the profile, which is itself due to the fact that the profile does not exactly follow the flowline and approaches the margin closely. However, for profile A, which follows the true direction of flow, the surface velocity increase is monotonic towards the grounding line, and the stress regime is very similar.

In the main trunk $(200-315 \mathrm{~km})$, where the surface slope is small and smooth, the driving stress is low $(10-40 \mathrm{kPa})$ and is mainly balanced by lateral and basal drag, and longitudinal stress gradients are rather small (Fig. 3c and h). Similar sections can be identified for the basal friction coefficient $\beta^{2}$ (Fig. $3 \mathrm{~d}$ and i). For profile $\mathrm{A}$, there is no significant difference in the value of $\beta^{2}$ between the steep and flat parts of the main trough, a result which is not obvious considering the huge differences in $\tau_{\mathrm{b}}$ and driving stress. For profile $\mathrm{B}, \beta^{2}$ is slightly 


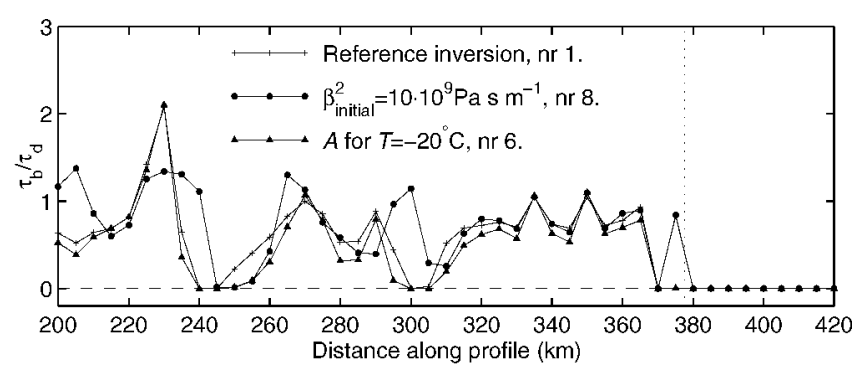

Fig. 4. Calculated basal traction relative to the driving stress along profile A, shown for the reference inversion (crosses), an inversion with a constant initial $\beta^{2}=10 \times 10^{9} \mathrm{~Pa} \mathrm{sm}^{-1}$ (dots) and with a rate factor corresponding to $-20^{\circ} \mathrm{C}$ (triangles). The numbers in the legend here and in Figures 5 and 6 indicate the inversion run number from Table 1.

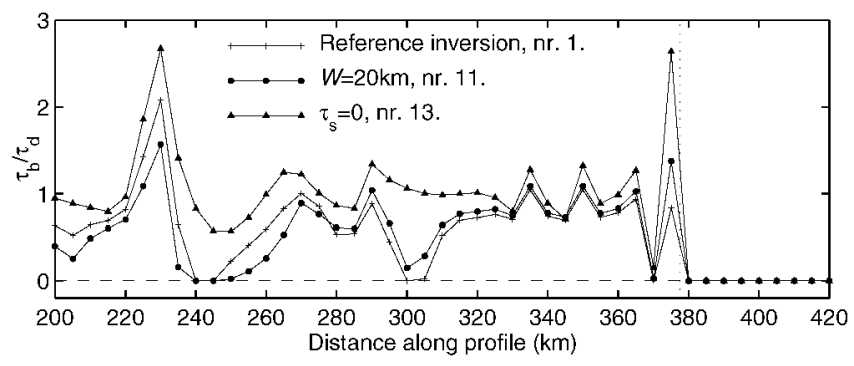

Fig. 5. Calculated basal traction relative to the driving stress along profile A, shown for the reference inversion (crosses), an inversion with a constant half-width $W=20 \mathrm{~km}$ (dots) and with no lateral drag $\left(\tau_{\mathrm{s}}=0\right)$ (triangles).

higher in the zone of high $\tau_{\mathrm{b}}$. This might be due to the velocity reduction where the profile comes too close to the margin.

\section{Additional model runs}

To check the sensitivity of the model inversion and parameter assumptions, additional model runs and inversions have been calculated by varying the rate factor $A$, the width $W$ of the ice stream, the shear softening factor $f_{\text {soft }}$ at the margins and the

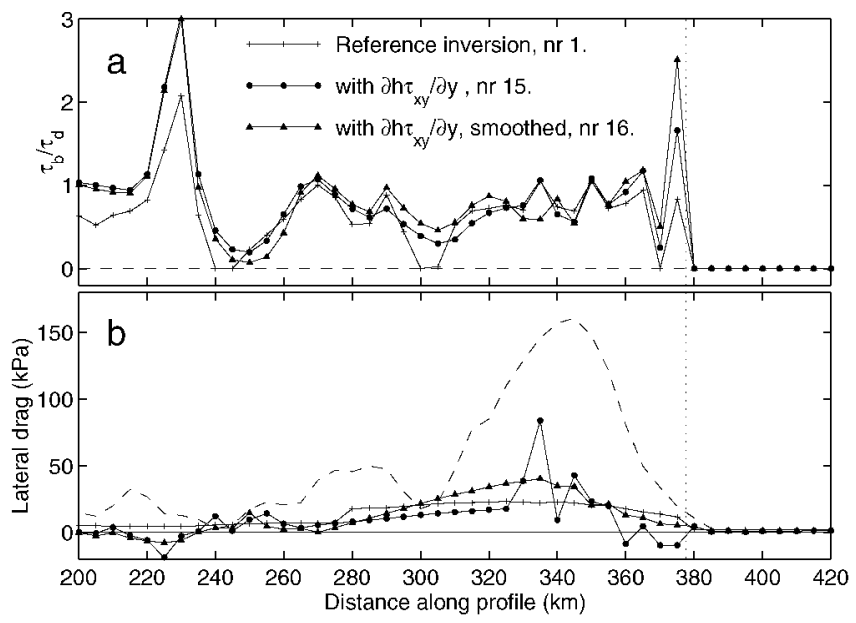

Fig. 6. (a) Calculated basal traction relative to the driving stress, and (b) lateral drag along profile A, shown for the reference inversion (crosses) and two inversions using a lateral drag calculated directly from the two-dimensional surface velocity field for two differently smoothed versions. The dashed line indicates the driving stress. initial guess for $\beta^{2}$ (Figs 4 and 5). Furthermore, inversion runs were performed with a model which uses calculated lateral drag determined directly from the observed two-dimensional surface velocity field (Fig. 6). An overview of the additional inversion runs is given in Table 1. In general, the stress regime looks very similar to the reference inversion discussed above. Where the driving stress is low, the effect of changes in width, rate factor $A$ or shear softening on the stress distribution is significant in relation to $\tau_{\mathrm{d}}$. In the region of high surface velocities $(x>200 \mathrm{~km}), \beta^{2}$ is not very sensitive to variations in the parameters and is rather low. This indicates that a small change in $\beta^{2}$ could affect the flow significantly.

\section{Sensitivity to the initial choice of $\beta^{2}$}

To check the influence of the initial guess for the model parameter $\beta^{2}$ on the final $\beta^{2}$ or on $\tau_{\mathrm{b}}$, additional inversions with a constant initial $\beta^{2}$ of $2.5 \times 10^{9}$ and $10.0 \times 10^{9} \mathrm{~Pa} \mathrm{~s} \mathrm{~m}^{-1}$, respectively, are calculated (Fig. 4). The resulting $\beta^{2}$ and $\tau_{\mathrm{b}}$ distributions are very similar, which suggests that the inversion method used is not significantly affected by the choice of the initial guess for $\beta^{2}$.

\section{Inversion for $\beta$ and the basal topography}

A run in which the model is inverted for the basal topography $b$ (in addition to $\beta$ ) has also been performed. The idea is that $b$ is not well constrained by measurements and is therefore taken as an additional unk nown model parameter. The observed basal topography $b_{\mathrm{d}}$ is used as initial guess for $b$. An additional term $J_{\mathrm{b}}$, which measures the misfit between observed and calculated bed elevation, is added to the total cost function $J$ which is now given by

$$
J=J_{u}+J_{\mathrm{b}}=\sum_{i=1}^{m} \frac{\left(u_{i}^{\mathrm{d}}-u_{i}\right)^{2}}{\sigma_{u}^{2}}+\sum_{i=1}^{m} \frac{\left(b_{i}^{\mathrm{d}}-b_{i}\right)^{2}}{\sigma_{\mathrm{b}}^{2}},
$$

where $\sigma_{\mathrm{b}}$ is the measurement error of the basal elevation which is here set to $10 \mathrm{~m}$. The gradient of $L$ is then given by $\nabla J=\left(\partial L / \partial \beta_{j}, \partial L / \partial b_{j}\right)$.

In our case, the contribution of $J_{\mathrm{b}}$ turns out to be rather small in comparison to $J_{u}$, and the final $\beta$ and $\tau_{\mathrm{b}}$ distributions are very similar to the reference inversion performed for $\beta$ only (Table 1). Thus, changes of $b$ in the range of the measurement error do not significantly affect the inverted $\beta$.

Lateral drag derived from the observed two-dimensional velocity field Model runs for both profiles have been performed which use an alternative way of including lateral drag in our icestream model. Using Glen's flow law, $\tau_{x y}$ is approximated as

$$
\tau_{x y}=\frac{1}{(2 A)^{1 / 3}}\left(\frac{\partial u}{\partial y}\right)^{1 / 3} .
$$

Using this expression the lateral shear stress term $\partial h \tau_{x y} / \partial y$ in Equation (1) can be explicitly calculated from the observed two-dimensional surface velocity field along the two profiles. The lateral drag calculated from the original velocity data is rather noisy because it is found from the second derivative of the velocity across the ice stream. Therefore, additional model inversions were performed using differently smoothed surface velocity fields to represent lateral drag (Table 1, Nos. 15-18.). The resulting lateral drag and the inverted basal drag for profile A are shown in Figure 6 and confirm the general stress pattern found above. The large differences of the basal drag relative to the driving stress behind the grounding line are mainly due to differences in the lateral drag. 
Table 1. List of the performed inversions that were accepted ( $J$ fell below 63.17). The mean values (overbar) along the transect and their standard deviations $(\sigma)$ are listed for the basal drag $\bar{\tau}_{\mathrm{b}}$, lateral drag $\overline{h / W}$ in $k P a$ and the basal friction coefficient $\overline{\beta^{2}}$ in Pa sm ${ }^{-1}$

\begin{tabular}{|c|c|c|c|c|c|c|c|c|}
\hline No. & Description & $J$ & $\overline{\mathrm{\tau}}_{\mathrm{b}}$ & $\sigma_{\tau_{\mathrm{b}}}$ & $\overline{h / W \tau_{\mathrm{s}}}$ & $\sigma_{h /} W \tau_{\mathrm{s}}$ & $\bar{\beta}^{2}$ & $\sigma_{\beta^{2}}$ \\
\hline 1 & Reference inversion, profile A & 33.2 & 39.4 & 41.9 & 13.5 & 7.5 & $3.2 \times 10^{9}$ & $3.4 \times 10^{9}$ \\
\hline 3 & $A=6.4 \times 10^{-24} \mathrm{~Pa}^{-3} \mathrm{~s}^{-1}, T=0^{\circ} \mathrm{C}$ & 8.6 & 47.3 & 44.6 & 5.6 & 3.1 & $3.9 \times 10^{9}$ & $3.8 \times 10^{9}$ \\
\hline 4 & $A=1.6 \times 10^{-24} \mathrm{~Pa}^{-3} \mathrm{~s}^{-1}, T=-5^{\circ} \mathrm{C}$ & 18.6 & 43.7 & 43.6 & 9.1 & 5.0 & $3.6 \times 10^{9}$ & $3.7 \times 10^{9}$ \\
\hline 5 & $A=2.9 \times 10^{-25} \mathrm{~Pa}^{-3} \mathrm{~s}^{-1}, T=-15^{\circ} \mathrm{C}$ & 35.0 & 36.9 & 41.5 & 16.1 & 8.9 & $3.1 \times 10^{9}$ & $3.6 \times 10^{9}$ \\
\hline 6 & $A=9.4 \times 10^{-26} \mathrm{~Pa}^{-3} \mathrm{~s}^{-1}, \mathrm{~T}=-20^{\circ} \mathrm{C}$ & 60.2 & 33.7 & 40.9 & 19.2 & 10.6 & $2.9 \times 10^{9}$ & $3.5 \times 10^{9}$ \\
\hline 7 & $\beta_{\text {initial }}^{2}=2.5 \times 10^{9} \mathrm{~Pa} \mathrm{~s} \mathrm{~m}^{-1}$ & 34.7 & 39.8 & 41.9 & 13.5 & 7.4 & $3.4 \times 10^{9}$ & $3.1 \times 10^{9}$ \\
\hline 8 & $\beta_{\text {initial }}^{2}=10.0 \times 10^{9} \mathrm{~Pa} \mathrm{~s} \mathrm{~m}^{-1}$ & 38.3 & 39.9 & 41.7 & 13.4 & 7.4 & $3.4 \times 10^{9}$ & $3.2 \times 10^{9}$ \\
\hline 9 & Shear-softening $f_{\text {soft }}=5$ & 28.5 & 45.1 & 43.8 & 7.9 & 4.4 & $3.8 \times 10^{9}$ & $3.7 \times 10^{9}$ \\
\hline 10 & Shear-softening $f_{\text {soft }}=20$ & 28.0 & 48.0 & 45.1 & 4.9 & 2.7 & $4.0 \times 10^{9}$ & $3.9 \times 10^{9}$ \\
\hline 11 & Width $W=20 \mathrm{~km}$ & 48.1 & 40.6 & 44.7 & 12.5 & 3.3 & $2.8 \times 10^{9}$ & $2.7 \times 10^{9}$ \\
\hline 12 & Width $W=30 \mathrm{~km}$ & 37.3 & 45.9 & 45.4 & 7.2 & 1.9 & $3.5 \times 10^{9}$ & $3.3 \times 10^{9}$ \\
\hline 13 & No lateral drag $\tau_{\mathrm{s}}=0$ & 23.7 & 53.4 & 48.2 & 0.0 & - & $4.5 \times 10^{9}$ & $4.2 \times 10^{9}$ \\
\hline 14 & Inversion $\beta$ and $b$ & 35.3 & 39.0 & 36.4 & 10.8 & 7.6 & $6.9 \times 10^{9}$ & $8.0 \times 10^{9}$ \\
\hline 16 & $\partial h \tau_{x y} / \partial y$ from $u_{\mathrm{d}}(x, y)$, smoothed, profile A & 41.5 & 40.2 & 37.0 & 12.8 & 14.4 & $3.9 \times 10^{9}$ & $4.4 \times 10^{9}$ \\
\hline 17 & $\partial h \tau_{x y} / \partial y$ from $u_{\mathrm{d}}(x, y)$, profile B & 54.3 & 44.8 & 46.0 & 5.9 & 8.4 & $2.6 \times 10^{9}$ & $2.0 \times 10^{9}$ \\
\hline 18 & $\partial h \tau_{x y} / \partial y$ from $u_{\mathrm{d}}(x, y)$, smoothed, profile B & 52.5 & 46.1 & 45.4 & 3.7 & 6.0 & $2.7 \times 10^{9}$ & $2.0 \times 10^{9}$ \\
\hline
\end{tabular}

Note: Unless otherwise indicated, the inversions were performed for profile A.

\section{DISCUSSION}

The control method used to invert for $\beta$ (and therefore $\tau_{\mathrm{b}}$ ), is based on the assumption that the flow model is correct. Our numerical flow model is, however, an approximation and therefore its limitations have to be taken into account. The main trunk $(200-365 \mathrm{~km})$ and the grounding-line region (365-385 km) of PIG are characterized by both low driving stress and basal traction. Here we are confident that the model assumption of zero vertical shear is legitimate. The area upstream of the grounding line $(315-365 \mathrm{~km})$ is characterized by both high driving stress $(150 \mathrm{kPa})$ and basal traction.

The assumption that the velocity is constant with depth will overestimate the importance of lateral drag and longitudinal stress gradients, simply because horizontal velocity gradients are smaller at depth if deformation is important. This means that our calculated basal drag will be underestimated. Accounting for the depth variation in velocity would increase the importance of basal drag but not significantly alter the conclusion that the basal drag is the main resistance to flow in this region of high driving stress.

Our a priori analysis indicated that significant vertical shear may occur in this area (based on the driving-stress consideration alone). Because we can now estimate all stress components, the validity of our a priori assumption for this area can be checked. In a similar manner to MacAyeal and others (1995), we compare basal traction (as opposed to driving stress) to the stress necessary to obtain the observed surface velocity by vertical shear alone. Our results indicate that basal traction is close to the driving stress in this area (Fig. 3). Our previous a priori analysis (Flow model section) is therefore supported by model results, and detailed modelling (full stress regime) is required for this area of PIG.

In the parameterization of Van der Veen and Whillans (1996), the resistance from the sides is expected to be overestimated, which is clearly indicated in the ice-shelf part by the fact that the calculated lateral drag exceeds the driving stress. Shear softening would also reduce the resistance from the sides. Additionally, lateral and basal drag are considered independently, which is appropriate if either lateral or basal drag balances most of the driving stress, but may be less realistic in regions where both are equally important.

There is no significant difference in the value of $\beta^{2}$ between the steep and flat parts of the main trough, although $\tau_{\mathrm{b}}$ is very different. Accepting our assumptions for the model and the relation at the basal boundary, the constant value of $\beta^{2}$ could be interpreted to mean that the slip properties at the bed-ice interface are very similar. This suggests that the peak in basal traction $\tau_{\mathrm{b}}$ is a consequence of the high driving stress in the region behind the grounding line. The low values of $\beta^{2}$ in the grounding-line region are consistent with a region of light grounding as suggested for the ice plain (Corr and others, 2001). The dynamics of the ice plain may therefore be significantly affected by a thinning in the region of the grounding line or ice shelf, as observed in the last few years (Shepherd and others, 2001).

\section{CONGLUSIONS}

By the use of a simple ice-stream model, a control method has been successfully applied to determine the basal friction distribution $\tau_{\mathrm{b}}$ along the transect of PIG from observed surface velocities. The high driving stress up-glacier from the grounding-line region is dominantly balanced by basal friction, which reaches values up to $150 \mathrm{kPa}$. In the main trough further up-glacier and in the grounding-line region, lateral drag and longitudinal stresses also become important.

The control method allows us to invert for other unknown or poorly constrained model parameters, as demonstrated for the basal topography, or to include additional or alternative observational constraints. The thinning rate of the surface $\partial s / \partial t$ can be measured by remote-sensing techniques with high accuracy (Shepherd and others, 2001) and could easily be included as constraint in the control algorithm by adding the misfit between its observed and modelled value in the cost function. However, it would also require the incorporation and knowledge of the basal geometry across the channel.

The control method hinges on the use of a numerical flow model. The main model assumption of no vertical shear of the horizontal velocity is found to be doubtful in 
the region of the driving-stress peak. Further, the parameterization of the lateral drag oversimplifies the resistance from the sides in the main trough and in the grounding-line region. An improved understanding of the stress regime and dynamics of PIG therefore requires the use of a flow model that incorporates vertical shear of the horizontal velocity and considers the flow in both horizontal dimensions, but this would require detailed knowledge of basal topography outside profiles A and B.

\section{AGKNOWLEDGEMENTS}

We thank A. Shepherd from University College London for providing the velocity data, and the British Antarctic Survey for allowing us to use the basal topography data. We further thank S. Coles for statistical support. C.J. van der Veen, C. Raymond and two anonymous reviewers made very useful comments that resulted in much improved manuscript. This work was supported by the grant NER/A/S/2000/00419 from U.K. Natural Environment Research Council, and the Centre for Polar Observation and Modelling.

\section{REFERENGES}

Corr, H. F. J., C. S. M. Doake, A. Jenkins and D. G. Vaughan. 2001. Investigations of an "ice plain" in the mouth of Pine Island Glacier, Antarctica. 7. Glaciol., 47 (156), 51-57.

Echelmeyer, K. A., W. D. Harrison, C. Larsen and J. E. Mitchell. 1994. The role of the margins in the dynamics of an active ice stream. 7 . Glaciol., 40(136), 527-538

Giovinetto, M. B., N. M. Waters and C. R. Bentley. 1990. Dependence of Antarctic surface mass balance on temperature, elevation, and distance to open ocean. 7. Geophys. Res., 95(D4), 3517-3531.

Glen, J.W. 1955. The creep of polycrystalline ice. Proc. R. Soc. London, Ser. A, 228(1175), 519-538.

Hutter, K. 1983. Theoretical glaciology; material science of ice and the mechanics of glaciers and ice sheets. Dordrecht, etc., D. Reidel Publishing Co.; Tokyo,
Terra Scientific Publishing Co.

MacAyeal, D. R. 1989. Large-scale ice flow over a viscous basal sediment: theory and application to Ice Stream B, Antarctica. 7. Geophys. Res., 94(B4), 4071-4087.

MacAyeal, D. R. 1993. A tutorial on the use of control methods in ice-sheet modeling. F. Glaciol., 39(131), 91-98.

MacAyeal, D. R., R. A. Bindschadler and T. A. Scambos. 1995. Basal friction of Ice Stream E, West Antarctica. 7. Glaciol., 41(138), 247-262.

Mendenhall, W., D. D. Wackerly and R. L. Scheaffer. 1989. Mathematical statistics with applications. Fourth edition. Belmont, CA, Duxbury Press.

Menke, W. 1989. Geophysical data analysis: discrete inverse theory. Revised edition. New York, etc., Academic Press.

Paterson, W. S. B. 1994. The physics of glaciers. Third edition. Oxford, etc., Elsevier.

Paterson, W. S. B. and W. F. Budd. 1982. Flow parameters for ice sheet modelling. Cold Reg. Sci. Technol., 6(2), 175-177.

Press, W. H., B. P. Flannery, S. A. Teukolsky and W. T. Vetterling. 1989. Numerical recipes; the art of scientific computing (Fortran version). Cambridge, Cambridge University Press.

Raymond, C. 1996. Shear margins in glaciers and ice sheets. f. Glaciol., 42(140), 90-102.

Rignot, E. J. 1998. Fast recession of a West Antarctic glacier. Science, 281 (5376), 549-551.

Schlitzer, R. 1993. Determining the mean, large-scale circulation of the Atlantic with the Adjoint Method. F. Phys. Oceanogr., 23(9), 1935-1952.

Shepherd, A., D. J. Wingham, J. A. D. Mansley and H. F. J. Corr. 2001 Inland thinning of Pine Island Glacier, West Antarctica. Science, 291(5505), 862-864.

Thacker,W. C. 1988. Fitting models to inadequate data by enforcing spatial and temporal smoothness. F. Geophys. Res., 93(C9), 10,655-10,665.

Thacker, W. C. and R. B. Long. 1988. Fitting dynamics to data. 7. Geophys. Res., 93(C2), 1227-1240.

Van der Veen, C. J. and I. M. Whillans. 1989. Force budget: I. Theory and numerical methods. F. Glaciol., 35(119), 53-60.

Van der Veen, C. J. and I. M. Whillans. 1996. Model experiments on the evolution and stability of ice streams. Ann. Glaciol., 23, 129-137.

Vaughan, D. G. and 9 others. 2001. A review of Pine Island Glacier basin, West Antarctica: hypotheses of instability vs. observations of change. In Alley, R. B. and R. A. Bindschadler, eds. The West Antarctic ice sheet: behavior and environment. Washington, DC, American Geophysical Union, 237-256. (Antarctic Research Series 77.)

Whillans, I. M., Y. H. Chen, C. J. van derVeen and T. J. Hughes. 1989. Force budget: III. Application to three-dimensional flow of Byrd Glacier, Antarctica. F. Glaciol., 35(119), 68-80. 